\title{
Successful surgical repair of transected common carotid artery after 12 hours from trauma
}

\author{
Grigol Keshelava $^{1}$, Zurab Robakidze ${ }^{1}$, Gela Kurashvili ${ }^{1}$, Irakli Goletiani ${ }^{2}$ \\ ${ }^{1}$ Department of Vascular Surgery, West Georgian National Centre of Interventional Medicine, 83 Javakishvili St, Kutaisi, Georgia 4600 \\ ${ }^{2}$ Department of Neurology, West Georgian National Centre of Interventional Medicine, 83 Javakishvili St, Kutaisi, Georgia 4600
}

\section{Email address:}

gaga_keshelava@yahoo.com (G. Keshelava)

\section{To cite this article:}

Grigol Keshelava, Zurab Robakidze, Gela Kurashvili, Irakli Goletiani. Successful Surgical Repair of Transected Common Carotid Artery after 12 Hours from Trauma. Journal of Surgery. Vol. 2, No. 5, 2014, pp. 65-67. doi: 10.11648/j.js.20140205.12

\begin{abstract}
This case reports a case of transection of common carotid artery (CCA) resulting in cerebral ischemia and stroke in 21-year-old-man. A patient was operated after 12 hours from penetrating trauma of CCA with favorable result. Hemiparesis disappeared on the fifth day, subsequently performing the surgery. Speech recovered within the month since the operation took place.
\end{abstract}

Keywords: Common Carotid Artery; Carotid bypass

\section{Introduction}

Major vascular injuries in the region of the neck are most frequently the result of penetrating trauma. Evaluation and management of patients with injury of the neck remains highly controversial. Most studies involve small number of patients with a lack of standardization of the nature of the injury in reporting outcome (1). The total transection of CCA is very rarely encountered.

\section{Case}

Case: A 21- year-old male patient was inflicted a wound on the neck by a shard. Being hospitalized to the regional clinic, he was unconscious. Regarding arterial blood pressure, it was $60 / 20$. As the patient was taken to the hospital, there did not seem to be symptoms of hemorrhage. Resuscitation treatment was conducted and after stabilization of hemodynamic, general surgeons fulfilled the wound revision and stitch. Eight hours later after the surgery, right sided hemiparesis, aphasia and somnolence were revealed as soon as the patient pulled through. He was taken to our hospital (10 hours later after the injury). By Triplex scan and CT angiography left CCA thrombosis was divulged. According to the brain CT scan ischemic insult and swelling in the left cerebral hemisphere was discovered (Fig. 1 a,b)

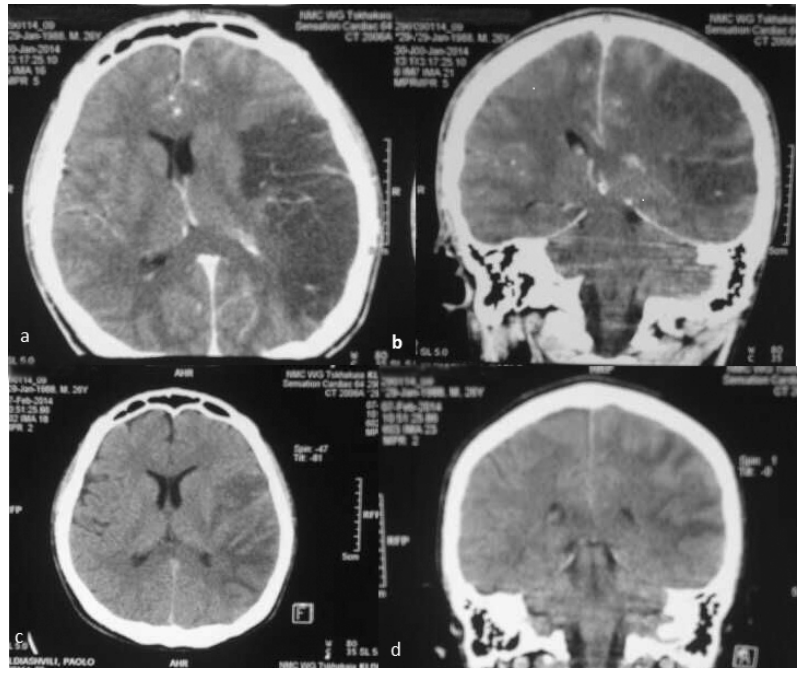

Fig 1. $a, b$ - CT scan after 10h from trauma shoved ischemic insult and edema of left hemisphere $c, d$-CT scan after 24 h from surgery shoved sharp regress of ischemic zone and edema of left hemisphere

The patient was taken in the operating block immediately. Approach to the left CCA was conducted. Intraoperatively was found out full transection of the CCA (Fig.2 a). Also, the crossed thrombosed ends. Checking of retrograde blood flow showed satisfactory results. Reconstructive surgery was decided. It was possible to establish integrity of common carotid artery through PTFE prosthesis without using temporary bypass (Fig. 2 b). 


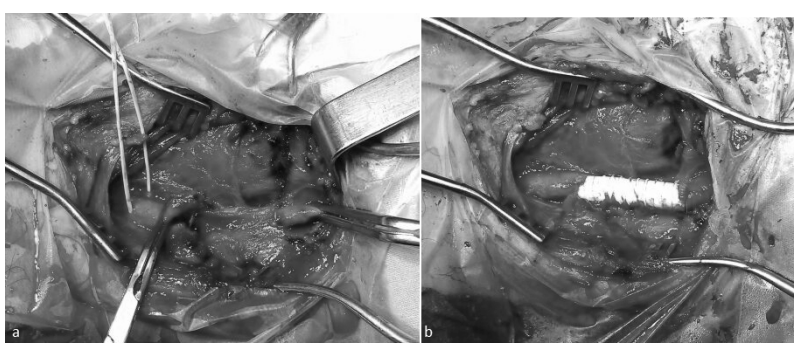

Fig 2. a-Full transection of left CCA, b-repair of left CCA with PTFE graft

It took 12 hours to recover integrity of common carotid artery from the injury.

After a while from the operation, the patient was provided with anticoagulant therapy included minimum dose in order to avoid transformation of ischemia into hemorrhage insult and Mannitol for brain edema.

Within 24 hours after the surgery, controlling computer tomography was held, which authenticated common carotid artery normal output and sharp regress of ischemia and brain edema (Fig.1 c,d). After 48 hours from the surgery, as the patient regained consciousness, he was interactive with adequate thinking. Right sided hemiparesis disappeared on the fifth day, subsequently performing the surgery. Speech recovered within the month since the operation took place. The patient was discharged from the medical center on day 14 after surgery. Acetylsalicylic acid was used as antipatelet therapy permanently (100mg/day). The 6-month postoperative followup visit revealed the normal condition of the patient. Duplexscanning revealed good permeability of vascular graft.

\section{Discussion}

The neck injuries are divided into three anatomical zones. Level I refers to the common carotid artery's intrathoracic segment at aortic arch. Level II spans the distance between the sternal notch and the Blaisdell line. Level III is the region superior to the Blaisdell line (2). Control of blood vessels in zone I and III is difficult. Zone II is the most common site of carotid artery injuries and standard surgical approach can be performed. For control of blood in I zone the sternotomy must be performed. In zone III, anatomical structures such as the distal part of the carotid artery, vertebral artery, the parotid gland, the pharynx, and cranial nerves IX, X, XI, XII are present (3).

In case in which a vascular injury is diagnosed, conservative treatment with anticoagulant drugs, surgical treatment such as vessel ligation or revascularization, and interventional procedures should be considered. In our case the retrograde blood flow showed satisfactory results and ligature of CCA may also cause fatal outcomes. Endovascular procedure was not able due to full transaction of CCA and reconstructive surgery was decided.

Ramadan et al. cited neurologic deficit as a result of damage to the extracranial cerebral vasculature was first reported in 1552 when Ambroise Pare ligated right CCA (4). Existence of retrograde blood flow is a very important indicator for reconstructive surgery of the traumatized carotid artery. Toit et al. (5). described the indications of carotid ligation. Arterial ligation should be considered only for distal arterial occlusion (absence of retrograde flow), unattainable distal control of internal carotid artery in III zone, and in the instable patient with multiple trauma.

The usual pathologic lesion following carotid artery tauma, is ischemic cerebral infarction caused by hypoperfusin or thromboembolism $(6,7,8)$. Study of Johnston et al. revealed possibility of prevention or reversion of cerebral ischemia by arterial repair in some patients (6).

In general, in diagnosing vascular injuries due to neck laceration, angiography is performed in cases of injury occurring in zone I and III. In case of injury in zone II the laceration site is confirmed through neck exploration (10). Kim et al (3) confirmed the priority of endovascular treatment for carotid artery injury; however endovascular procedure was not able due to full transaction of CCA. Our article reports a case of successful surgical repair of completely transected CCA after 12 hours from trauma. We could not find a similar case in the literature. At present, it can be concluded that penetrating injury to the common carotid or internal carotid artery should be repaired rather than ligated when technically possible and when retrograde arterial flow is established. Subsequent ischemic or hemorrhagic cerebral infarction is unpredictable, but the overall outcome is superior to that with ligation of the injured artery. However, the carotid artery should be ligated in comatose patients, critical unstable patients or difficult-to-repair lesions $(9,11)$. For traumatic distal internal carotid artery occlusion in neurologically intact patients, it should be managed by anticoagulation alone or as adjunctive ligation (5). Stent-graft treatment is considered in selected patients with a proximal lesion (requiring sternotomy) or distal internal carotid artery injury. The bleeding, intraluminal clot, compression symptoms caused by hematoma, concomittent injuries needing exploration, and infected wounds as relative contraindications (5). This therapeutic method may in future be more extensively applied in selected patients, especially with newly developed adjunctive neurologic protection devices $(11,12)$.

\section{Conclusion}

Penetrating injury of CCA should be repaired rather than ligated when technically possible. Operative repair offers the best chance of recovery.

\section{References}

[1] Mittal VK, Paulson TJ, Colaiuta E, Habib FA, Penney DG, Daly B, Young SC. Carotid artery injuries and their management. J Cardiovasc Surg (torino) 2000; 41(3): 423-431.

[2] Feliciano DV. Management of penetrating injuries to carotid artery. World J Surg 2001; 25(8): 1028- 1035.

[3] Kim JP, Park JJ, Won SJ, Woo SH. Penetrating carotid artery injuries treated by an urgent endovascular stent technique: report of two cases. Chonnam Med J 2011; 47: 134.137. 
[4] Ramadan F, Rutledge R, Oller D, Howell P, Baker C, Keagy B. Carotid artery trauma: a review of contemporary trauma center experiences. J Vasc Surg 1995; 21: 46-55.

[5] Toit DF, van Schalkwyk GD, Wadee SA, Warren BL. Neurologic outcome after penetrating extracranial arterial trauma. J Vasc Surg 2003; 38: 257-262.

[6] Johnston RHJ, Wall MJJ, Mattox KL. Innominate artery trauma: a thirty-year experience. J Vasc Surg 1993; 17: 134139.

[7] Meyer JS, Denny-Brown D. The cerebral collateral circulation. I: factors influencing collateral blood flow. Neurol 1957; 7: 447-458.

[8] Denny-Brown D, Meyer JS. The cerebral collateral circulation. II: production of cerebral infarction by ischemic anoxia and its reversibility in early stages. Neurol 1957; 7: 567-578.
[9] Navsaria P, Omoshoro-Jones J, Nicol A. An analysis of 32 surgically managed penetrating carotid artery injuries. Eur J Vasc Endovasc Surg 2002; 24(4): 349-355.

[10] Perry MO, Snyder WH, Thal ER. Carotid artery injuries caused by blunt trauma. Ann Surg 1980; 192: 74-77.

[11] Grego F, Frigatti P, Amista P, Lepidi S, Antonella M, Carrolo $\mathrm{C}$ et al. Prospective comparative study of two cerebral protection devices in carotid angioplasty and stenting. J Cardiovasc Surg (Torino) 2002; 43: 391-397.

[12] Whitlow PL, Lyklyk P, Londero H, Mendiz OA, Mathias K et al. Carotid stenting protected with an emboli containment system. Stroke 2002; 33: 1308-1314. 